\title{
Emergent, Situated and Prospective Ethics for Child-Computer Interaction Research
}

\author{
Alissa N. Antle \\ SIAT, Simon Fraser University \\ Surrey, B.C., Canada \\ aantle@sfu.ca \\ Christopher Frauenberger \\ HCI Group, TU Wien \\ Vienna, Austria \\ christopher.frauenberger \\ @tuwien.ac.at

\section{Monica Landoni} \\ Informatics, USI, Technology \\ Lugano, Switzerland \\ monica.landoni@usi.ch \\ Jerry Alan Fails \\ CS, Bosie State University \\ Boise, ID, USA \\ jerryfails@boisestate.edu
}

Permission to make digital or hard copies of part or all of this work for personal or classroon use is granted without fee provided that copies are not made or distributed for profit or commercial advantage and that copies bear this notice and the full citation on the first page. Copyrights for third-party components of this work must be honored. For all other uses, contact the owner/author(s).

IDC 2020 Extended Abstracts, June 21-24, 2020, London, UK

(C) 2020 Copyright is held by the owner/author(s).

ACM ISBN 978-1-4503-8020-1/20/06

DOI: https://doi.org/10.1145/3397617.339805

\section{Marina Jirotka}

CS, University of Oxford

Oxford, England, UK

marina.jirotka@cs.ox.ac.uk

Helena Webb

CS, University of Oxford

Oxford, England, UK

helena.webb@cs.ox.ac.uk

Nalin Tutiyaphuengprasert

Darunsikkhalai School for

Innovative Learning

King Mongkut's University of

Technology,

Thonburi, Bangkok, Thailand

nalin.tt19@gmail.com

\begin{abstract}
The increasing presence of interactive technologies in children's lives poses critical ethical questions for researchers and designers. Discourse specific to these intersecting topics is nascent, but is spread across communities and largely developed retrospectively.

This workshop brings together those interested in ethical issues arising when researching, designing, and deploying technologies for children. The focus is on exploring approaches that are emergent and situated, arising during research or after deployment. Workshop activities will include: exploring ethical themes emerging in $\mathrm{HCI}$ research for children; synthesizing and adapting current applicable ethical guidance; identifying gaps; and developing preliminary methods and guidance to address these gaps. Outcomes will extend current best practices in ethics in ways that promote children's protection, empowerment and wellbeing.
\end{abstract}

\section{Author Keywords}

Ethics; children; youth; in-action ethics; prospective ethics; child-computer interaction; vulnerable populations; cultural sensitivity; ethical guidance.

\section{ACM Classification Keywords}

Social and professional topics $\rightarrow$ Codes of ethics. 


\section{Background}

From its advent, computer technology has transformed our world more than any other force [13]. In 2010, the Kaiser Family Foundation reported that Generation M2, that is children ${ }^{1}$ (8-18 years), spend about 7.5 hours a day using digital media [19] and has since increased. In industrialized nations this generation grew up with no memory of life before the internet and smart phones [16] and this digital revolution increasingly impacts children's everyday lives in developing countries. For example, UNICEF found that $40 \%$ of Vietnamese children in rural areas used the internet for educational purposes [25], and likely many more have access through family members' cellular phones.

Through design, research and development, the $\mathrm{HCI}$ community is linked to children's use of interactive technologies. The speed of innovation in research combined with the heightened importance of involving children as participants in research (e.g. $[4,5,22]$ ) raises longstanding and emergent ethical concerns that may not be known to the HCI community [8].

Children's rights to participate and be protected in research has been guided by declarations for over 50 years (e.g. United Nations Declaration of the Rights of the Child [1959]; Declaration of Helsinki [1964]; United Nations Convention on the Rights of the Child [1989]) [11]. Yet research in emerging interactive technologies creates new ethical scenarios that cannot be addressed using existing guidance. Consider the case in which a wearable EEG-based mobile neurofeedback app is developed to help young children living in poverty learn to self-regulate anxiety and attention in order to

\footnotetext{
${ }^{1}$ As per the the UN Convention on the Rights of the Child, children
} refers to all legal minors below the age of 18 years old. improve educational outcomes [3]. The case raises longstanding issues around social justice and children's rights to participate, such as: How to adequately get assent from a population that cannot fully understand the risks or benefits of research into this new form of technology? More importantly, the case raises new questions that move beyond available guidance. For example: How does these children's interaction with neurodata, which represents internal brain states that may or may not be modifiable, impact (both positively and negatively) their sense of agency, identity, and epistemic authority? Addressing such questions requires methods that focus on the in-situ exploration of impacts [10] and consideration of trade-offs between children's rights to protection, participation and provision [20]. What is needed is guidance that can help researchers both anticipate future issues and impacts of these new classes of technologies, as well as be sensitive to unforeseen emergent issues.

As the research community expands to include researchers from a broader spectrum of nations and cultures, and as researchers work with children from diverse populations, other underexplored issues emerge from the cultural and philosophical lenses that we as researchers have with regards to science, technology and children, either implicitly or explicitly, when we plan and implement research [27]). For example in [7], Frauenberger makes a compelling case for a critical realist conceptualization of disability (e.g. autism) that ... "serves the project as a guiding principle in planning and conducting workshops or studies in an ethically and morally responsible way." Scholars of childhood also urge for the consideration of how children's interactions with new technologies are firmly located in the wider settings of cultural and social processes: the 
institutional organization of childhood, schooling, family, and the different uses of private and public space $[16,21]$. It is imperative that we develop ethics guidance that is sensitive to the (multiple) cultures of childhood and does not assume either a technodeterminist or utopian vision of the future.

It is clear that ethics in HCI for children cannot keep up with the speed in which new ethical scenarios emerge, often in situ [10]. Yet over the last few years there has been rising awareness in the $\mathrm{CHI}$ community through keynotes, panels, and SIGs highlighting the importance of proactively considering ethical issues and moral perspectives in $\mathrm{HCI}$ for children (e.g. $[1,8,9,15])$. The challenge to address ethics in $\mathrm{HCI}$ is shared with the rest of the HCI research community - as the CHI 2018 roundtable indicated in terms of its goals ... "the development of community norms for research ethics in the face of evolving technology and methods." We argue that the time is right for this workshop - it is imperative to conduct research and develop guidance purposefully and prospectively; leading to the establishment of community norms based on actionable and situation-sensitive guidance for those creating interactive technologies with vulnerable populations, such as children. Our motivation for this workshop is this need for a holistic, situated, culturally sensitive, and value driven approach to ethics, as part of what has been called the third paradigm in $\mathrm{HCI}[10,14]$.

This workshop is the first formal gathering to address these challenges within the broader HCI community. In alignment with the SIGCHI Ethics Committee our aim is to facilitate discussion around research ethics within the community, yet drawing on previous works in other communities (e.g. $[6,11,17,21])$. We seek to bring together individuals who work closely with children in various contexts through research, design, and deployment of interactive technologies in both industrialized and developing countries. Specifically, our goals are to:

- Bring forward and discuss cases that reveal new ethical challenges that may arise during research and after deployment;

- Identify ethical priorities (see Themes below) to direct the development of a research agenda in ethics of $\mathrm{HCI}$ and children;

- Identify and adapt existing guidance and begin to develop new guidance where possible;

- Highlight the role values, culture and philosophical perspectives play in developing guidance;

- Formalize a preliminary working group with members from $\mathrm{HCI}$ and other concerned communities;

- Commit to the dissemination of results (e.g. ACM Interactions, special issue of IJCCI on Ethics).

\section{Themes}

Our themes build on issues, opportunities and ethical questions that were identified in the authors' previous works as well as through previous panels, SIGs, and roundtables. The following preliminary set of themes are those we hope to address:

Benefits of Co-Design and Evaluation Participation $\mathrm{HCI}$ researchers who work with children in design or evaluation processes have rarely focused on ethical aspects of their work (see $[22,28]$ for exceptions). In [2], Antle raises a fundamental question - rarely addressed openly - which is: what do children gain from participating in our research and what constitutes 
evidence of those benefits? Despite the Interaction Design for Children (IDC) Conference requirement for authors to include a section discussing ethics (e.g. selection and participation), most IDC papers cite formal ethical procedures designed to assess risk such as those required by many ethics board applications (which vary across countries: e.g. IRB - USA, REB Canada, or no formal ethics committee in parts of Europe). Following from [20], we see a need to educate and encourage researchers to examine and document the trade-offs between children's protection and participation alongside provision and benefit in these discussions. In parallel, we see a need to move beyond static, formal ethics processes, which do little to guide situated ethical dilemmas that arise and judgements that must be made during research [10]. For example, in a mental health study young children quickly became strongly attached to a smart toy prototype; requiring researchers to reframe the deployment narrative and change the target age for subsequent research [23].

\section{Data, Privacy and Security}

In a recent CHI SIG [15], participants discussed and debated how ubiquitous computing and big data are impacting the lives of children. Questions arose including: What are the ethical issues of children's rights to control and own data about themselves? How does that change as they age? How does the information that is tracked affect children's values? While some guidance can be taken from medical ethics, the impact of private technology companies versus health authorities complicates this one among the many issues that have entered the discourse around data, privacy and security (e.g. [28]) and must be addressed relative to children.
Identity, Agency, and Authority

Research has shown that technological artifacts, like medical devices (e.g. glasses, inhalers) that predate interactive technologies play an important part in the construction of identity [16]. Ethnographic studies in schools suggest these objects can both have an impact on their context of use and be modified by the complex layers of practice encountered there. Both participation in research and use of new technologies can impact children's sense of who they are [12], raising ethical questions such as, what is the impact of big data that effectively quantifies children and impacts on selfperception and epistemic authority? Or the previously mentioned question, how might neurodata impact a child's sense of agency [17]?

Equity, Access and Social Justice

Another important theme involves social justice, which requires community norms that ensure that children who may most benefit are included in research even if they are difficult to access or work with [1]. This again raises the need for ethical guidance, which may be adapted from non-HCI sources (e.g. [26]) but must also be responsive to situational specific factors. For example, in one work in sensitive settings conducting activities with marginalized children, the authors were confronted with moral dilemmas in real time, which were not foreseen in formal ethical reviews and required them to make judgements on the spot, which may contradict overarching ethical principles [24].

Impact of Researcher's Philosophical Stance A researcher's philosophical position, and associated values, whether explicit or implicit, includes assumptions about how they view and value science and technology (e.g. beneficial, neutral, deterministic). 
This in turn impacts how they view their research and/or design problem and how they think about the impact of their work on children. As a result, their stance forms a foundation for what they see or do not see as ethical issues. We include this theme in order to highlight the philosophical foundations of ethical issues rather than focus merely on the pragmatics of ethics.

\section{Organizers}

The organizers represent different disciplinary backgrounds, cultures, and experiences of conducting $\mathrm{HCI}$ research with children, and each has experience chairing, organizing and/or participating in previous ACM workshops (e.g. CHI, IDC, CSCW).

Alissa N. Antle is a professor, innovator and designer, whose research pushes the boundaries of computation to explore how technical innovations can improve, augment, and support children's development.

Christopher Frauenberger is a Senior Researcher at the Human-Computer Interaction Group, TU Wien. He designs technology with and for marginalized user groups, in particular autistic children.

Monica Landoni is a Senior Researcher in the Faculty of Informatics at Università della Svizzera Italiana. She has worked on national and European projects investigating technology to support children when searching, writing and reading.

Jerry Alan Fails is an Associate Professor in Computer Science at Boise State University. He focuses on HCI and participatory design, with interest in connecting children with one another, getting them active, and encouraging real-world exploration.
Marina Jirotka is Professor of Human-Centred Computing at the University of Oxford. She investigates technologies that are more responsive to societal acceptability - recently awarded an EPSRC Established Career Fellowship focused on Developing Responsible Robots for the Digital Economy.

Nalin Tutiyaphuengprasert is the co-founder and senior vice provost of Darunsikkhalai School for Innovative Learning in Thailand. She has worked in schools with teachers and students on constructionismbased projects including DSIL FabLearn in conjunction with Stanford University.

Helena Webb is a Senior Researcher of HumanCentred Computing at the University of Oxford. Her projects explore contextual interaction with and innovation of technologies by focusing on impact of setting and social action. Projects include: Digital Wildfire, UnBias, LabHack, and RoboTIPS project.

\section{Link to Website}

The workshop website will include a description, schedule, preparation, and organizers. We will post selected position papers and suggest readings including relevant ethical discourse and cases from $\mathrm{HCI}$ and other disciplines (e.g. $[10,11,17,18,20,22,24])$.

URL: antle.iat.sfu.ca/chi-2020-workshop

\section{Pre-Workshop Plans}

This workshop will accommodate 10-25 participants. Before the workshop potential participants will submit a 2-page position paper in the form of a mini-case study related to their experiences with workshop issues, themes and goals. We will distribute the CfP to HCI mailing lists (e.g. CHI Announcements, CHI-Kids, the active Child-Computer Interaction IFIP and Facebook 
groups, and social media) and reach out to childfocused ethics research centers involving (e.g. Oxford Internet Institute, UNICEF-IRC) and industry representatives (e.g. Sesame Workshop, Lego, Google). The position papers will be screened by the organizers for clarity and to ensure we have multicultural perspectives. We will solicit input into a shared annotated bibliography (AB) of children's ethics before the workshop. This will direct some of the workshop discussion. Participants are expected to read all accepted submissions and the $A B$ in advance.

\section{Workshop Structure}

This one-day workshop is split into six sections. The first section (45 minutes) includes introductions of participants, an explanation of workshop themes (including new ones that might arise from position papers) and a review of ethical stances and philosophical worldviews from other communities on the influence of science and technology. The second section (45 minutes) includes group activities for deeper exploration of the existing (or new) themes focused on balancing protection, participation and provision, and asks participants to consider what is known (retrospective) and what we need to know (prospective). After a morning coffee break, the third activity (60 minutes) involves a whole group session to share and prioritize issues raised. After lunch there will be two sequential activities separated by a coffee break (45 minutes each). In these working groups participants will discuss relevant guidance within a prioritized area. Where no guidance exists, groups will generate considerations and questions that must be addressed. We will repeat this activity so participants can join two working groups. During the final section (60-75 minutes and carrying on over dinner), the whole group will come together to share, weave together and record our findings.

\section{Post-Workshop Plans}

Workshop outcomes will include a revised annotated bibliography with statements of best practices and a research agenda for the community. The workshop serves to create a preliminary working group and a network for attendees to access when ethical issues arise in future work. Follow-up action items include identification of opportunities for a symposium to address unanswered questions raised in the workshop. Outcomes for the broader community will include a plain language report hosted on the website. The organizers will work with the editorial board of the International Journal of Child-Computer Interaction (Antle, Frauenberger and Landoni are on the Editorial Board) to create a special issue on ethics in $\mathrm{HCI}$ for children and youth. In addition we will create a forum for ACM Interactions similar to $[2,15]$.

\section{Call for Participation (250 words)}

The increasing role of interactive technologies in children's lives, poses critical ethical questions for the creators of these technologies. Despite many concerns, ethical guidance is largely lacking. This workshop brings together researchers, designers, psychologists,

educators, and industry representatives who work with and for children. This one-day workshop has the following goals: (1) Bring forward and discuss cases that reveal new ethical challenges; (2) Identify a research agenda to address gaps where existing governance and guidance does not apply; (3) Reveal the role cultural and philosophical perspectives play in developing guidance; (4) Develop preliminary actionable guidance that focuses on emergent, situated 
and prospective guidance that can inform research practice; (5) Formalize a preliminary working group.

Participants are invited to submit a 2 page position paper in the SIGCHI Extended Abstracts format. Papers should take the form of a mini-case study related to experiences with the workshop focus, themes and goals. Our themes provide inspiration for authors to think past existing ethical issues to consider emergent and unique aspects of ethics in $\mathrm{HCI}$ for children. We welcome the suggestion of new themes in the position papers. We strongly urge workshop applicants to explicitly state their cultural and philosophical stance.

The organizing committee will select submissions based on the quality and diversity of the contribution. Please send your submission by May 152020 to

aantle@sfu.ca. URL: antle.iat.sfu.ca/chi-2020workshop. One author from each accepted paper must register for the workshop and one day at $\mathrm{CHI}$.

\section{References}

1. Alissa N. Antle. 2017. Crazy like us: Design for vulnerable populations. In Proc. Conf. on Interaction Design and Children (IDC '17), 3-4.

2. Alissa N. Antle. 2017. The ethics of doing research with vulnerable populations. Interactions 24, 6: 74-77.

3. Alissa N. Antle, Leslie Chesick, Srilekha K. Sridharan, and Emily Cramer. 2018. East meets west: A mobile brain-computer system that helps children living in poverty learn to self-regulate. Personal and Ubiquitous Computing: 1-28.

4. Laura Benton and Hilary Johnson. 2015. Widening participation in technology design: A review of the involvement of children with special educational needs and disabilities. Int. J. of Child-Comp. Int. 3-4: 23-40.
5. Jerry Alan Fails, Mona Leigh Guha, and Allison Druin. 2013. Methods and Techniques for Involving Children in the Design of New Technology for Children. Found. and Trends in Human-Computer Interaction 6, 2: 85-166.

6. Casey Fiesler, Jeff Hancock, Amy Bruckman, Michael Muller, Cosmin Munteanu, and Melissa Densmore. 2018. Disability and Technology. In Ext. Abs.Conf. on Human Factors in Computing Systems (CHI EA '18), panel05.

7. Christopher Frauenberger. 2015. Disability and technology: A critical realist perspective. In Pro. Conf. Computers \& Accessibility (ASSETS '15), 89-96. h

8. Christopher Frauenberger, Alissa N. Antle, Monica Landoni, Janet C. Read, and Jerry Alan Fails. 2018. Ethics in interaction design and children: A panel and community dialogue. In Pro. Conf. on Interaction Design and Children (IDC '18), 748-752.

9. Christopher Frauenberger, Monica Landoni, Jerry Alan Fails, Janet C. Read, Alissa N. Antle, and Pauline Gourlet. 2019. Broadening the Discussion of Ethics in the Interaction Design and Children Community. In Proc. Conf. on Interaction Design and Children, 3-7.

10.Christopher Frauenberger, Marjo Rauhala, and Geraldine Fitzpatrick. 2017. In-action ethics. Interacting with Computers 29, 2: 220-236.

11.Urs Gasser and Sandra Cortesi. 2016. Children's rights and digital technologies: Introduction to the discourse and some meta-observations. Handbook of Children's Rights. M. Ruck, M. Peterson-Badali \& M. Freeman, eds., Taylor \& Francis.

12.A. E. Guttmacher. 2011. Invited address: A vision for the future of child development research.

13. Yuval Noah Harari. 2018. 21 Lessons for the 21st Century. Spiegel \& Grau, New York.

14.Steve Harrison, Phoebe Sengers, and Deborah Tatar. 2011. Making epistemological trouble: Third-paradigm 
$\mathrm{HCI}$ as successor science. Interacting with Computers 23, 5: 385-392.

15. Hourcade, Juan Pablo, Anthony, L., Fails, J., Iversen, O.S., Rubegni, E., Skov, M., Slovak, P., Walsh, G., and Zeising, A. 2018. SIG Report back to the community: Child computer interaction, ubiquitous technologies and big data. ACM Interactions, November + December.

16.Ian Hutchby and Jo Moran-Ellis. 2013. Children, Technology and Culture: The Impacts of Technologies in Children's Everyday Lives. Routledge.

17.Judy Illes, Alissa Antle, Hayami Lou, Holly Longstaff, Vasiliki Rahimzadeh, Patrick J McDonald, and H F Machiel Van der Loos. 2019. Involving children with neurodevelopmental disorders in biomedical research. The Lancet Child \& Adolescent Health 3, 3: 143-144.

18.UNICEF Office of Research- Innocenti. Ethical Research for Children. UNICEF-IRC. Retrieved from unicefirc.org/article/1809-ethical-research-for-children.htm

19. Kaiser Family Foundation. 2010. Daily Media Use Among Children and Teens Up Dramatically From Five Years Ago. Daily Media Use Among Children and Teens Up Dramatically From Five Years Ago. Retrieved from kff.org/disparities-policy/press-release/daily-mediause-among-children-and-teens-up-dramatically-fromfive-years-ago/

20.Eva Lievens, Sonia Livingstone, Sharon McLaughlin Brian O'Neill, and Valerie Verdoodt. 2018. Children's Rights and Digital Technologies. In International Human Rights of Children, Ursula Kilkelly and Ton Liefaard (eds.). Springer Singapore, Singapore, 1-27.

21.Sonia Livingstone, Giovanna Mascheroni, and Elisabeth Staksrud. 2018. European research on children's internet use: assessing the past and anticipating the future. New Media and Society 20: 1103-1122.

22.Brenna McNally, Mona Leigh Guha, Matthew Louis Mauriello, and Allison Druin. 2016. Children's
Perspectives on Ethical Issues Surrounding Their Past Involvement on a Participatory Design Team. In Proc. Conf. on Human Factors in Computing Systems, 35953606.

23.Petr Slovak, Nikki Theofanopoulou, Alessia Cecchet, Peter Cottrell, Altarriba Bertran, Ella Dagan, Julian Childs, and Katherine Isbister. 2018. "I Just Let Him Cry. . . ": Designing Socio-Technical. In Int. Conf. on Computer Supported Collaborative Work and Social Computing.

24.Katta Spiel, Emeline Brulé, Christopherher Frauenberger, Gilles Bailly, and Geraldine Fitzpatrick. Micro-Ethics For Participatory Design With Marginalised Children. In Proc. Participatory Design Conference: Full Papers - Volume 1 (PDC '18), 12 pages.

25.Unicef Report. At a Glance: Vietnam. Retrieved October 7, 2018 from unicef.org/infobycountry/vietnam_statistics.htm

26.2016. What We Know about Ethical Research Involving Children in Humanitarian Settings: An Overview of Principles, the Literature and Case Studies.

27. Ethical Research Involving Children, ERIC, Child Ethics. Ethical Research Involving Children. Retrieved from childethics.com/

28.SOUPS 2018 Workshop Report: Designing Privacy and Security Tools for Children and Teenagers Privacy Education and Research Lab (PEARL). Retrieved from pearl.umd.edu/2018/10/soups-2018workshop-report-designing-privacy-and-securitytools-for-children-and-teenagers/ 